\title{
DETECCIÓN DE DIFICULTADES EN EL PROCESO DE APRENDIZAJE DEL CONCEPTO DE SIMETRÍA EN EDUCACIÓN INFANTIL
}

\section{DETECTION OF DIFFICULTIES IN THE LEARNING PROCESS OF THE CONCEPT OF SYMMETRY IN EARLY CHILDHOOD EDUCATION}

\author{
Ainhoa Berciano \\ Universidad del País Vasco/Euskal Herriko Unibertsitatea - España \\ ainhoa.berciano@ehu.eus \\ Clara Jiménez-Gestal \\ Universidad de la Rioja - España \\ clara.jimenez@unirioja.es \\ María Salgado \\ Universidade de Santiago de Compostela - España \\ maria.salgado@usc.es
}

\section{Resumen}

Cuando se habla de simetría, esta se entiende como un concepto no solo matemático, sino también asociado a la belleza, o como evidencia de un equilibrio en procesos de la naturaleza. En este sentido, es claro que la comprensión de dicho concepto está ligada con ciertas tareas asociadas a la capacidad de visualización; por lo que en este artículo pretendemos indagar en las dificultades que muestran los niños y las niñas de 5 años de educación infantil en los distintos niveles de comprensión de la simetría. Para tal fin, se ha implementado una secuencia de aprendizaje basada en la matemática contextualizada, específicamente diseñada para fomentar la comprensión del concepto de simetría, para posteriormente realizar un estudio de casos. Los resultados nos llevan a concluir que la mayor dificultad surge cuando los niños y las niñas se enfrentan a la detección de los distintos ejes de simetría, en los casos en que hay más de uno.

Palabras clave: simetría, dificultades, Educación Infantil, visualización, matemática contextualizada

\begin{abstract}
When we talk about symmetry, it is understood not only as a mathematical concept, but also as a concept associated with beauty, or as evidence of balance in natural processes. In this sense, it is clear that the understanding of this concept is linked to certain tasks associated with the ability to visualize. Therefore, in this article we intend to investigate the difficulties shown by 5-year-old
\end{abstract}


children in the different levels of understanding symmetry. To this end, we have implemented a learning sequence specifically designed to promote the understanding of the concept of symmetry and based on contextualized mathematics, to subsequently carry out a case study. The results lead us to conclude that the greatest difficulty arises when children face the detection of different axes of symmetry, when there is more than one.

Keywords: symmetry, difficulties, early childhood education, visualisation, contextualised maths

\section{INTRODUCCIÓN}

La educación matemática en las primeras edades es un ámbito de investigación en auge. Prueba de ello es el gran número de publicaciones realizadas en el marco de congresos internacionales como el Congress of European Research in Mathematics Education (CERME), más concretamente en el grupo Early Years Mathematics (EYM); auspiciadas por la Federación Iberoamericana de Sociedades de Educación Matemática (FISEM) o por la National Council of Teachers of Mathematics (NCTM); o en un ámbito más local en el Grupo de Investigación en Educación Matemática (IEMI) de la Sociedad Española de Investigación en Educación Matemática (SEIEM). Uno de los aspectos señalados en las agendas de investigación en educación matemática infantil se refiere a la construcción del conocimiento matemático, cómo se desarrollan los contenidos y los procesos matemáticos y las conexiones entre ellos (ALSINA, 2019; NOVO; BERCIANO; ALSINA, 2019).

La mayoría de las niñas y los niños cuando comienza su etapa escolar ya poseen muchas capacidades matemáticas y les gusta utilizarlas. No es raro observarles, tanto en clase como en su vida cotidiana, hacer estimaciones de cantidades, comparaciones de figuras o tamaños, agrupaciones o repartos, en lo que se podría considerar manifestaciones de la matemática informal, incluso en edades de 0 a 3 años (ALSINA; BERCIANO, 2020). Sin embargo, para gran parte de la población adulta las matemáticas suponen un recuerdo incómodo de su etapa escolar. Estudios como el de Hidalgo et al. (2013) describen cómo evolucionan las creencias acerca de las matemáticas en estudiantes de los distintos niveles educativos. Este cambio en la percepción de las matemáticas a lo largo de la vida refuerza la necesidad de indagar sobre el modo en que el conocimiento matemático se produce.

En este sentido se enfocan las recomendaciones de las actuales propuestas curriculares, con la incorporación de la noción competencia matemática definida como la capacidad para entender las matemáticas y aplicarlas a contextos de la vida cotidiana (OCDE, 2017), o como la habilidad que permite interpretar la información del entorno, 
tener un conocimiento más amplio de la realidad y resolver problemas aplicables a cualquier circunstancia.

Además, la National Council of Teachers of Mathematics (NCTM, 2003) señala de modo explícito para esta etapa (Pre-K-2, que abarca de los 3 hasta los 8 años) la necesidad de trabajar tareas directamente relacionadas con el conocimiento del entorno y el espacio, que están estrechamente vinculadas con la capacidad de visualización, habilidad que tienen las personas para "realizar ciertas tareas que requieren «ver» 0 «imaginar» mentalmente los objetos geométricos espaciales, así como relacionar los objetos y realizar determinadas operaciones o transformaciones geométricas con los mismos" (GODINO; GONZATO; CAJARAVILLE; FERNANDEZ, 2012).

Para desarrollar esta capacidad, la NCTM hace hincapié en la necesidad de trabajar las transformaciones en el espacio y, en particular, en la simetría. Así, los contenidos que se trabajen en esta etapa deben favorecer que los niños y las niñas sean capaces de "Reconocer y aplicar traslaciones, reflexiones y giros. Reconocer y crear formas que tengan simetrias".

Actualmente, para el caso de España, la Orden ECI/3963/2007, de 19 de diciembre, por la que se establece el currículo y se regula la ordenación de la educación infantil (MINISTERIO DE EDUCACIÓN Y CIENCIA, 2008) aunque no habla de competencias sino de habilidades matemáticas, manifiesta en la misma línea que se deben trabajar contenidos que hacen referencia a las nociones espaciales básicas y a la identificación de formas planas y tridimensionales, aspectos claramente relacionados con la visualización.

Finalmente, la importancia que tanto las organizaciones internacionales como la legislación nacional dan a la simetría junto con su presencia en diversos ámbitos de la vida, el arte y la naturaleza es lo que nos lleva a investigar acerca de las dificultades que la adquisición de este concepto supone en las primeras edades.

En este trabajo presentamos los resultados de un estudio cualitativo en el que se analizan las dificultades observadas en niños y niñas de 5 años de educación infantil en los distintos niveles de comprensión de la simetría, en el transcurso de una secuencia de aprendizaje específicamente diseñada para fomentar la comprensión del concepto y desarrollada en un contexto cotidiano. 
Berciano, A.; Jiménez-Gestal, C.; Salgado, M.

\section{FUNDAMENTACIÓN TEÓRICA}

\section{Experimento de enseñanza}

Un experimento de enseñanza es una secuencia de actividades diseñadas específicamente para ser llevadas al aula con el objetivo de recopilar información sobre el proceso de adquisición del conocimiento matemático por parte del alumnado. En el desarrollo de un experimento de enseñanza se establecen tres fases, en las que Molina, Castro, Molina y Castro (2011) identifican diferentes acciones.

En la primera fase, preparación del experimento, se definen tanto el objetivo de investigación como los objetivos instruccionales, se evalúan los conocimientos previos del alumnado y se diseña la secuencia de actividades que se van a realizar, acordes a la metodología de enseñanza elegida. Además, es necesario establecer qué datos se van a recoger y de qué modo.

La segunda fase es la experimentación. En esta fase se producen las intervenciones en el aula previamente a cada una de las cuales hay que identificar los objetivos instruccionales, diseñar la actuación de docentes e investigadoras y su interacción con el alumnado y establecer las hipótesis acerca de los resultados esperados. Durante las intervenciones se realiza la recogida de datos y las modificaciones oportunas sobre el diseño establecido si es necesario, y tras las mismas el análisis de los datos recogidos y la reformulación de las hipótesis cuando es preciso.

La tercera y última fase del experimento de enseñanza es el análisis retrospectivo de los datos que consiste esencialmente en recopilar y organizar toda la información recogida para su análisis.

Trabajos previos como Bernabeu, Moreno y Llinares (2019) muestran la eficacia de los experimentos de enseñanza para realizar investigaciones relacionadas con la investigación matemática en contextos de aula.

\section{Matemáticas en Educación Infantil}

La National Council of Teachers of Mathematics (NCTM, 2003), publica en el año 2000 los Principios y Estándares para la Educación Matemática, donde se da un alto valor a la matemática en la etapa referida a la educación infantil, en la que, partiendo del interés y curiosidad del alumnado por su entorno, propone el uso de metodologías que, por medio de la experimentación, fomenten la adquisición de contenidos y destrezas. Una matemática 
en la que los estándares de contenido y los estándares de proceso se trabajen conjuntamente. Este cambio de enfoque en el aprendizaje de las matemáticas revela la importancia de los procesos de comunicación como parte fundamental del aprendizaje. Tanto la comunicación entre iguales como la que se establece entre docente y discentes contribuyen a la adquisición de los contenidos y al desarrollo de las capacidades necesarias para considerar que la competencia matemática alcanza su desarrollo.

Así mismo, las orientaciones metodológicas del Ministerio de Educación y Ciencia (2008) indican que "los métodos de trabajo en ambos ciclos se basarán en las experiencias" (Art. 2. 4.) y que "Los contenidos de la Educación Infantil se abordarán por medio de propuestas integradas que tengan interés y sean significativas” (Art. 5. 4.).

La influencia de este tipo de metodologías en el aprendizaje de las matemáticas desde las primeras edades está ampliamente documentada. Ya en la década de los 90, Freudenthal (1991) avanzaba que niñas y niños deben enfrentarse a situaciones contextualizadas en entornos conocidos para adquirir paulatinamente los conocimientos matemáticos, desde los más concretos a los más abstractos.

\section{Geometría y simetría en Educación Infantil}

Así, de entre los aspectos matemáticamente más relevantes en la etapa infantil se encuentra el tratamiento de la geometría y su enseñanza, ya que resulta de suma importancia fomentar que los niños y niñas comprendan su entorno y lo dominen; sean capaces de orientarse en él; y adquieran, al mismo tiempo, ideas iniciales sobre el espacio a través de su propio esquema corporal.

En este contexto, el aprendizaje de la geometría no puede quedar relegado al reconocimiento de formas o sus nombres, sino que cuando hablamos de trabajar la geometría en edades tempranas, seguimos la línea de Canals (1997) cuando dice que solamente son objeto de la geometría los elementos del espacio que se refieren a la posición - relaciones espaciales, distancias y ángulos -, las formas - reconocimiento, construcción y categorización - y los cambios de posición y forma, a lo que llama "fenómenos geométricos". Este conocimiento incluye aspectos como el razonamiento espacial con el que debemos ser capaces de argumentar y resolver problemas relacionados con la posición, las formas de los objetos o transformaciones de los mismos, entre otros. Asumimos, de igual modo, que este conocimiento no comienza en la escuela, sino que viene definido por 
el entorno que nos rodea, por lo que su tratamiento escolar debe abordar de modo global el condicionamiento que niños y niñas tienen por medio del entorno físico que les envuelve.

Dentro de este conocimiento incipiente del espacio y de los conceptos matemáticos que están involucrados, en este artículo centramos nuestro interés en la simetría. En este sentido, la NCTM (2003) señala para esta etapa (Pre-K-2, que abarca de los 3 hasta los 8 años) contenidos relacionados directamente con las transformaciones en el espacio y en particular con la simetría: "Reconocer y aplicar traslaciones, reflexiones y giros. Reconocer y crear formas que tengan simetrías".

Ya hace cincuenta años, investigadores como Vurpillot (1976) hablaban de las nociones intuitivas de simetría detectadas desde las primeras edades, en el sentido de que no solo se prefieren los estímulos simétricos, sino que también se detectan más rápidamente, se discriminan con mayor precisión y a menudo se recuerdan mejor que los asimétricos. En este sentido, la preferencia por la simetría vertical se desarrolla entre los 4 y los 12 meses de edad (BORNSTEIN; FERDINANDSEN; GROSS, 1981) y la simetría bilateral vertical sigue siendo más fácil de manejar que la simetría horizontal, aunque muchos conceptos de simetría no están firmemente establecidos antes de los 12 años (GENKINS, 1975).

Por otro lado, los niños muy pequeños son capaces de crear diseños con simetría lineal y rotacional artísticos con materiales manipulativos y ya en K-2 pueden aprender a dibujar la otra mitad de una figura geométrica para crear una figura simétrica e identificar líneas de simetría (CLEMENTS, 2004, p.42).

Conforme a las investigaciones anteriormente detalladas, la comprensión de la simetría se puede descomponer en distintos niveles de adquisición atendiendo a los logros realizados por las niñas y los niños. Hemos recogido esta clasificación en la tabla 1 .

Tabla 1 - Niveles de comprensión de la geometría y descripción de logros

\begin{tabular}{|l|l|}
\hline Nivel & Descripción \\
\hline Descubrimiento & $\begin{array}{l}\text { Reconocimiento de objetos simétricos. } \\
\text { Preferencia de un objeto simétrico frene a otro que no lo es. }\end{array}$ \\
\hline Generación & $\begin{array}{l}\text { Creación de diseños con simetría con materiales manipulativos. } \\
\text { Dibujo de la otra mitad de una figura geométrica. }\end{array}$ \\
\hline Justificación & $\begin{array}{l}\text { Explicación de por qué un objeto es simétrico. } \\
\text { Explicitación de los ejes de simetría. }\end{array}$ \\
\hline
\end{tabular}


Igualmente, otras investigaciones analizan las destrezas mostradas por niñas y niños cuando se plantean distintas tareas en el aula. Así, Sarama ha notado que los niños a menudo utilizan y se refieren a la simetría rotacional tanto como a la simetría lineal en el trabajo con bloques de patrones (SARAMA; CLEMENTS; VUKELIC, 1996). Clements (2004, p.276) y Blanco, Gorgal y Salgado (2018) proponen como primer acercamiento docente a dicho concepto en el aula de educación primaria el uso de figuras simétricas y ejes de simetría en figuras planas y tridimensionales; y en Giménez y Vanegas (2019) aparecen sugerencias de fenómenos y situaciones adecuados para trabajar las transformaciones, y en concreto la simetría.

En esta misma línea, investigaciones previas afirman que la correcta intervención docente sobre la simetría desde el comienzo favorece la no adquisición de errores persistentes en la edad adulta y el aprendizaje de la geometría (ACUÑA; MARTÍNEZ, 2013); y el tratamiento de la simetría resulta vital en la adquisición de habilidades de visualización porque, entre otros aspectos, favorece los procesos de razonamiento mediante la composición y descomposición (SÁMUEL; VANEGAS; GIMÉNEZ, 2016).

Aun así, a pesar de las recomendaciones de incorporar la simetría a la labor docente de aula en la educación infantil debido a sus bondades; según Clements (2004, p. 290), no hay un planteamiento curricular que potencie desarrollar la capacidad espacial asociada a la simetría, usando como base las intuiciones de los niños y las niñas, preferencia e interés en la simetría.

\section{METODOLOGÍA}

Para dar respuesta al objetivo descrito, esto es, indagar las dificultades que presentan los niños y las niñas de educación infantil en los distintos niveles de comprensión de la simetría, se ha desarrollado una investigación cualitativa, desde un paradigma interpretativo. En ella se han analizado los tipos de dificultades que niñas y niños muestran en su discurso cuando tratan de explicarse oralmente en diversas tareas de iniciación al concepto de simetría y sus propiedades.

Para la experimentación de campo, se ha optado por la realización de un experimento de enseñanza debido a que esta metodología de investigación permite a las investigadoras, además de diseñar las actividades de enseñanza, participar activamente 
durante la fase de experimentación en el desarrollo de las dinámicas de aula y posibilita el ajuste de la secuencia si es preciso.

El diseño del experimento se ha realizado acorde con las fases descritas en Molina, Castro, Molina y Castro (2011) y se ha tenido en cuenta el tipo de actividad que se podría desarrollar en el aula de educación infantil de modo estructurado, para lo que se han revisado las ya mencionadas investigaciones enfocadas en describir las dificultades y destrezas que niñas y niños de distintas edades muestran. En lo que continúa detallamos la fase 1 y la fase 2 del experimento de enseñanza.

\section{Fase 1: preparación del experimento.}

\section{Objetivos}

El problema de investigación consistente en detectar las dificultades que muestran los niños y niñas en los distintos niveles de comprensión de la simetría se puede concretar en tres objetivos:

1.- Detectar las dificultades surgidas en el proceso de descubrimiento y generación de simetrías.

2.- Caracterizar las dificultades que aparecen en la identificación de diferentes ejes de simetría.

3.- Analizar las dificultades que se presentan en la justificación de la simetría.

\section{Participantes}

La investigación se ha llevado a cabo con un total de 19 niños y niñas de 5 años en el último curso de educación infantil del C.E.I.P. Sigüeiro (A Coruña), en el marco de su actividad cotidiana de clase. Una de las investigadoras es su profesora habitual y la clase está habituada a recibir visitas y compartir con ellas sus tareas cotidianas.

\section{Diseño de la secuencia de actividades}

Las actividades han sido diseñadas conjuntamente por las autoras de este artículo, atendiendo a las orientaciones de Alsina (2016) para el diseño de actividades matemáticas competenciales: matematización del contexto, trabajo previo en el aula, trabajo en contexto, trabajo posterior en el aula y formalización de los aprendizajes adquiridos; y posteriormente son ellas quienes las han implementado en el propio centro. Es relevante 
destacar la importancia del contexto elegido para la realización de actividades matemáticas en educación infantil (SALGADO; BERCIANO; JIMÉNEZ-GESTAL, 2018; JIMÉNEZGESTAL; BERCIANO; SALGADO, 2019).

Para este diseño de actividades se parte de un cuento, ya conocido por la clase, a partir del cual se diseñan las tareas. Un aspecto que tienen en común todas las tareas es su carácter motivador y vivencial, bien sea por incitar al movimiento del propio cuerpo, bien por el uso de materiales que llaman la atención como espejos o pintura de dedos, o bien por la posibilidad de interacción y discusión con toda la clase.

La Tabla 2 recoge la secuencia de actividades completa, que se compone de 9. El fin de todas las actividades ha sido el mismo, avanzar en la construcción y comprensión de la noción de simetría a partir de acciones y elementos del entorno, si bien se han enfocado en tres aspectos diferenciados asociados a los niveles de comprensión de las simetrías (ver tabla 1): por un lado, actividades de descubrimiento o generación de simetrías, por otro, actividades de identificación de diferentes ejes de simetría y por otro, actividades de justificación de la simetría.

\section{Instrumentos de recogida de datos}

El desarrollo de las actividades se ha grabado en vídeo para su posterior transcripción y análisis por parte de las investigadoras. Paralelamente, se han analizado las producciones en papel de niñas y niños de las actividades 4, 5, 6 y 7 .

Además, para facilitar el análisis de la información se ha hecho hincapié en los hitos y las dificultades descritas en investigaciones previas, en el marco teórico. 
Tabla 2 - Secuencia de actividades

\begin{tabular}{|c|c|c|c|c|}
\hline Actividad & Descripción de la actividad & Objetivo instruccional & \begin{tabular}{|l|} 
Orientaciones (Alsina, \\
2016)
\end{tabular} & \begin{tabular}{|l|l|}
$\begin{array}{l}\text { Niveles } \\
\text { comprensión }\end{array}$ \\
\end{tabular} \\
\hline $\begin{array}{l}\text { Actividad 1: } \\
\text { Lectura del } \\
\text { cuento }\end{array}$ & $\begin{array}{l}\text { En gran grupo escucha del cuento ¿A qué sabe la luna? } \\
\text { Al finalizar, se realiza una asamblea, con el propósito } \\
\text { de reflexionar sobre el suceso final, la aparición de una } \\
\text { luna en el agua. }\end{array}$ & $\begin{array}{l}\text { Contextualización } \\
\text { Descubrimiento de simetrías }\end{array}$ & $\begin{array}{l}\text { Trabajo previo en el } \\
\text { aula } \\
\text { Trabajo en contexto }\end{array}$ & Descubrimiento \\
\hline $\begin{array}{l}\text { Actividad 2: } \\
\text { Mi cuerpo: el } \\
\text { espejo }\end{array}$ & $\begin{array}{l}\text { Se divide el aula en parejas y se reparten roles (espejo } \\
\text { o niño). A través de imitación, el espejo tendrá que } \\
\text { repetir acciones del niño. }\end{array}$ & Generación de simetrías & Trabajo en contexto & Generación \\
\hline $\begin{array}{l}\text { Actividad 3: } \\
\text { Nuestra cara: } \\
\text { autorretrato }\end{array}$ & $\begin{array}{l}\text { En un folio que contiene media cara del niño, con un } \\
\text { espejo, tratará de componer su propia cara. }\end{array}$ & $\begin{array}{l}\text { Descubrimiento } \\
\text { generación de simetrías }\end{array}$ & $\begin{array}{l}\text { Trabajo posterior en el } \\
\text { aula }\end{array}$ & Descubrimiento \\
\hline $\begin{array}{l}\text { Actividad 4: } \\
\text { Magia: ta, ta, } \\
\text { chan }\end{array}$ & $\begin{array}{l}\text { En un folio que contiene la silueta de media pera, } \\
\text { primero la pintarán con pintura de dedos, plegarán por } \\
\text { la mitad (el eje de simetría marcado) y a continuación } \\
\text { abrirán y descubrirán lo que aparece. }\end{array}$ & $\begin{array}{|ll|}\begin{array}{l}\text { Descubrimiento } \\
\text { generación de simetrías }\end{array} & \mathrm{y} \\
\end{array}$ & $\begin{array}{l}\text { Trabajo posterior en el } \\
\text { aula }\end{array}$ & Descubrimiento \\
\hline $\begin{array}{l}\text { Actividad 5: } \\
\text { Dibujo } \\
\text { simétrico }\end{array}$ & $\begin{array}{l}\text { Completar figuras simétricas dadas (media luna, o } \\
\text { medio pez, o media tortuga) con la ayuda de un espejo. }\end{array}$ & Gen & $\begin{array}{l}\text { Trabajo posterior en el } \\
\text { aula }\end{array}$ & Generación \\
\hline $\begin{array}{l}\text { Actividad 6: } \\
\text { Entrevista }\end{array}$ & $\begin{array}{l}\text { Proceso de reflexión y autoevaluación de la situación } \\
\text { 5. Para esta situación se emplea un espejo de } \\
\text { metacrilato. }\end{array}$ & $\begin{array}{l}\text { Descubrimiento de simetrías } \\
\text { Justificación de la simetría }\end{array}$ & $\begin{array}{l}\text { Formalización de los } \\
\text { aprendizajes adquiridos }\end{array}$ & $\begin{array}{l}\text { Descubrimiento } \\
\text { Justificación }\end{array}$ \\
\hline $\begin{array}{l}\text { Actividad 7: } \\
\text { Me sabe a... }\end{array}$ & $\begin{array}{l}\text { A través de formas tridimensionales (alimentos reales), } \\
\text { manipulan, exploran y descubren el plano o ejes de } \\
\text { simetría. }\end{array}$ & \begin{tabular}{|l|l|}
$\begin{array}{l}\text { Identificación de ejes de } \\
\text { simetría }\end{array}$ \\
\end{tabular} & $\begin{array}{l}\text { Formalización de los } \\
\text { aprendizajes adquiridos }\end{array}$ & Justificación \\
\hline $\begin{array}{l}\text { Actividad 8: } \\
\text { Gustómetro }\end{array}$ & $\begin{array}{l}\text { Degustación, valoración y representación en un } \\
\text { diagrama de sus preferencias. }\end{array}$ & \begin{tabular}{|l}
$\begin{array}{l}\text { Establecimiento de cone- } \\
\text { xiones intra-matemáticas. }\end{array}$ \\
\end{tabular} & & \\
\hline $\begin{array}{l}\text { Actividad 9: } \\
\text { Ilustración } \\
\text { cuento }\end{array}$ & $\begin{array}{l}\text { Representación de la escena final del cuento, con la } \\
\text { ilustración de todos los personajes, seriándolos en el } \\
\text { orden en que aparecen. }\end{array}$ & $\begin{array}{l}\text { Establecimiento de } \\
\text { conexiones con otras áreas. }\end{array}$ & $\begin{array}{l}\text { Formalización de los } \\
\text { aprendizajes adquiridos }\end{array}$ & \\
\hline
\end{tabular}




\section{Fase 2: experimentación}

La experimentación se ha llevado a cabo en dos jornadas consecutivas, con una duración total de $5 \mathrm{~h}$.

La primera sesión ha constado de 5 actividades, todas ellas dirigidas a trabajar el descubrimiento y generación de simetrías. La jornada ha comenzado con una actividad de contextualización, orientada a indagar sobre los conocimientos del concepto de simetría de que disponen los niños y las niñas. A continuación, se han realizado tres actividades en las que tienen que realizar un movimiento simétrico, explorar la simetría mediante el uso de espejos y hacer aparecer por arte de magia (y de pintura de dedos) la mitad de un objeto mediante el plegado de folios. La última actividad de la jornada ha consistido en dibujar la mitad simétrica que falta en una figura. Tras la primera sesión, las investigadoras han analizado el desarrollo de la misma y realizado los ajustes necesarios para la segunda.

Las propuestas para el segundo día han comenzado con una actividad de autoevaluación, en la que hay que comprobar si el dibujo realizado la víspera es simétrico o no, con la correspondiente justificación por parte de quien lo ha realizado. En la siguiente actividad, la observación y manipulación de algunos alimentos ha permitido identificar y descubrir uno o más ejes de simetría, estableciendo una relación entre las figuras tridimensionales y su representación plana. Finalmente, se han trabajado dos actividades de cierre de actividad, en las que se han establecido conexiones con otras áreas, tanto matemáticas como ajenas a la matemática.

\section{RESULTADOS}

En este apartado mostramos los resultados acerca de las dificultades que presentan los niños y las niñas de educación infantil en los distintos niveles de comprensión de la simetría, que se corresponde con la tercera fase del experimento de enseñanza, análisis retrospectivo de los datos. Para ello, en primer lugar, describimos cada una de las actividades realizadas y, a continuación, mostramos el análisis de las interacciones del niño o niña con la maestra en el desarrollo de las mismas. En este punto debemos mencionar que, con el fin de hacer más comprensible el artículo, hemos seleccionado 6 casos para mostrar los fragmentos de diálogo en distintas fases de la actividad (1 caso para la actividad 3, ídem en actividades 4, 5 y 6; y 3 casos para la actividad 7). Posteriormente, se presenta 
un resumen de los hallazgos más relevantes asociados a cada objetivo de investigación.

\section{Actividad 3. Nuestra cara: autorretrato}

Cada estudiante tiene en papel media cara propia y un espejo doble. A través de la interacción libre y la exploración con el espejo, descubre la simetría presente en las propiedades intrínsecas del rostro (2 ojos, 2 orejas, ...) (Figura 1 izquierda) y juega a duplicar, triplicar, cuadruplicar,... otras partes y/o rostro (Figura 1 derecha).

Figura 1 izquierda - Exploración de la simetría del rostro; Figura 1 derecha - Juego de espejos

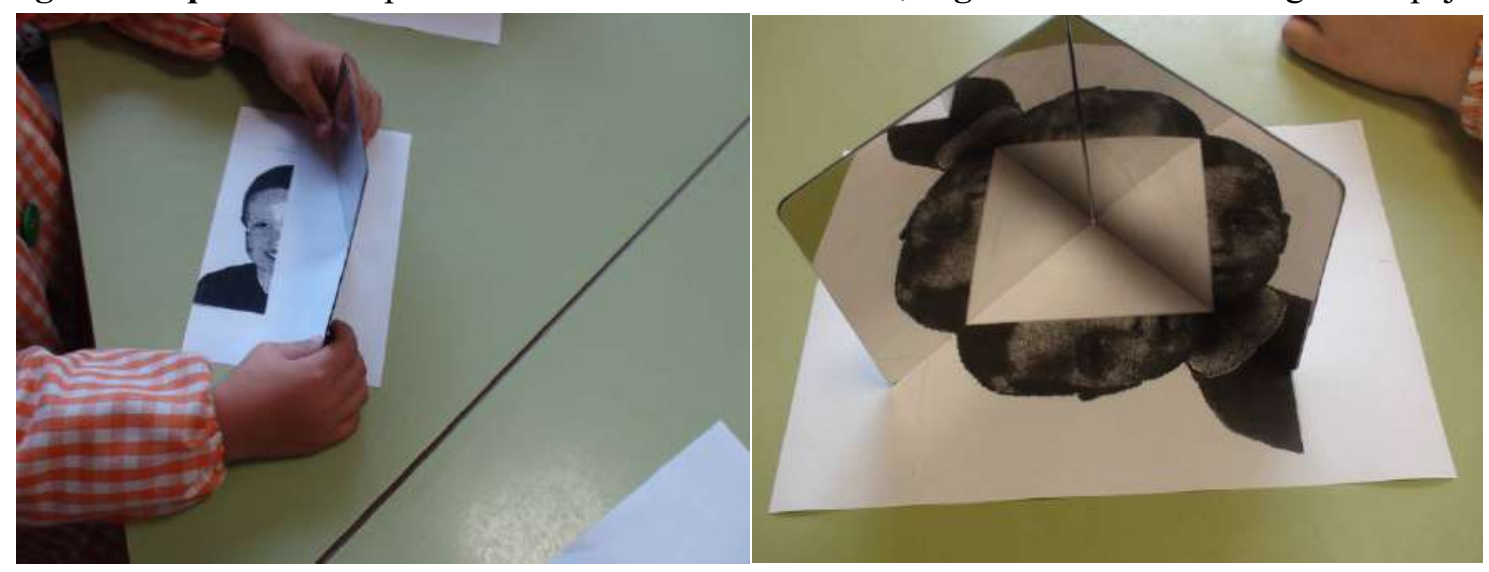

Fuente: archivo de la investigación

Tabla 3 - Transcripción del diálogo en la actividad 3

\begin{tabular}{|l|l|}
\hline 1 & Inv2: ¿Qué hay aquí, NoIk? \\
2 & Nolk: Dos caras iguales. \\
3 & $\begin{array}{r}\text { Inv1: Podéis probar en distintos sitios distintos reflejos. } \\
\text { Si lo abres más o menos, también salen distintas cosas. ¿No es así? } \\
4\end{array}$ \\
& Nolk: ¿Una tijera? En mi cara (Figura 2). \\
\hline
\end{tabular}

En el extracto de diálogo descrito en la Tabla 3 se aprecia como el alumno es capaz de descubrir la simetría en su rostro - “dos caras iguales” en (2) - aunque todavía no usa la palabra exacta, y cómo es capaz de transformar la realidad a través de la exploración, de componer y descomponer, obteniendo nuevos objetos - “¿Una tijera? En mi cara” en (4).

En esta actividad todos los niños y las niñas fueron capaces de identificar la simetría y la no simetría mediante la experimentación con diferentes posiciones de los espejos, aunque desde un punto de vista visual, sin argumentar acerca de la igualdad de distancias al eje de los elementos del objeto situados en posiciones simétricas.

\section{Actividad 4. Magia}

A partir del diálogo que se establece acerca de las preferencias y gustos alimenticios 
de los animales del cuento que nos ha servido de contextualización, ¿A qué sabe la luna?, se llega a que a la tortuga le sabe a pera y esto nos sirve para realizar la actividad.

Se facilita a cada estudiante la silueta de media pera dibujada en una hoja de papel. Con pintura de dedos se le pide que pinte la media silueta para, acto seguido doblar el papel por la mitad (Figura 2 izquierda). Tras presionar el papel y pronunciar las palabras mágicas "TA, TA, CHAN", al desplegar la hoja (Figura 2 derecha) descubrirá la pera completa.

Figura 2 izquierda - Plegado de papel; Figura 2 derecha - Desplegado de la hoja

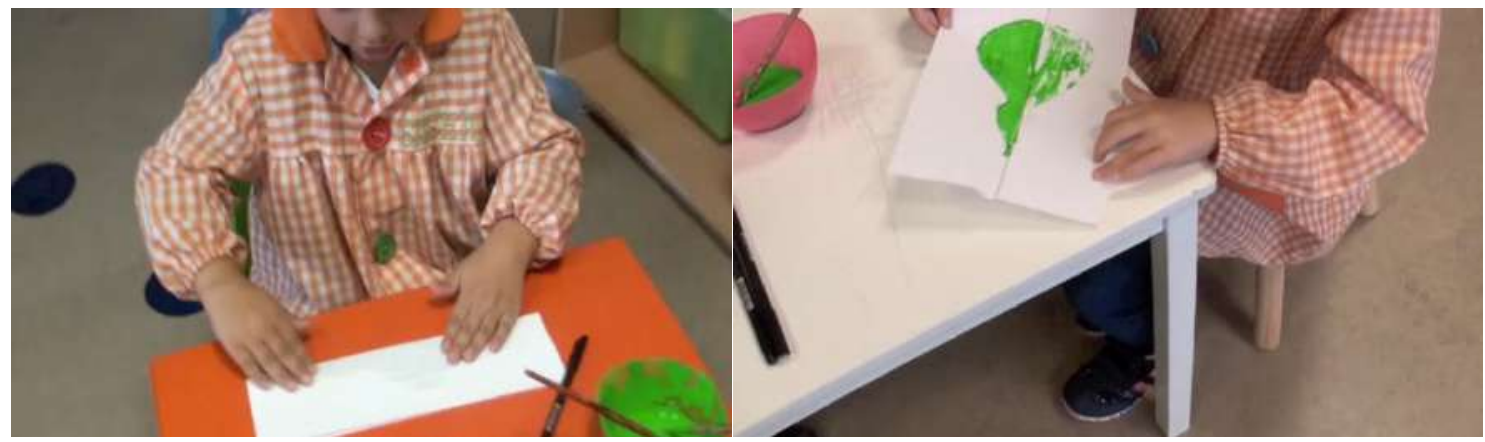

Fuente: archivo de la investigación

De este modo el alumnado tiene la oportunidad de intuir primero y descubrir después el eje de simetría de la pera.

Tabla 4 - Transcripción de diálogo en la actividad 4

\begin{tabular}{|l|l|}
\hline 1 & Inv2: Y, ¿cómo hiciste para tener una pera completa? \\
2 & NaY: Pintando. \\
3 & Inv2: ¿Pintando?, pero ¿cómo lo hiciste? \\
4 & NaY: Pinté la mitad y doblé, y después ya está. \\
\hline
\end{tabular}

La generación de la simetría es, en este caso, el resultado de la acción de plegado. No se ve como algo que el niño o la niña haya hecho conscientemente, sino que surge del truco de magia. Lo que sí es consciente es el reconocimiento de la simetría, ya que en los casos en que el marcado no es completo piden la pintura de nuevo para repetir el proceso.

\section{Actividades 5 y 6. Dibujo y entrevista}

Para la realización de la actividad 5 se ofrecen tres medias siluetas (de una tortuga, de una luna y de un pez) de figuras con forma simétrica. Cada estudiante elige una y a continuación, con la ayuda de un espejo, trata de completar la silueta lo mejor posible. En el desarrollo de la actividad, las investigadoras observaron como niñas y niños usaban los espejos para ver cómo debía quedar el dibujo y trataban de reproducir la línea observada. 
La actividad 6 se lleva a cabo el segundo día de trabajo de manera individual. Una de las investigadoras formula a cada niño o niña preguntas acerca del dibujo que hizo la víspera. Mediante un espejo de metacrilato se comprueba si el dibujo realizado coincide con la imagen simétrica y si no coincide se le pide que marque la línea que se ve con el espejo, se quita el espejo y se le pregunta ¿Qué ocurre?

Tabla 5 - Diálogo en la actividad 6

\begin{tabular}{|c|l|}
\hline 1 & Inv1: ¿Qué hay aquí? \\
2 & NoY: Una luna. \\
3 & Inv1: ¿Cómo lo hiciste?, ¿la dibujaste tú? \\
5 & NoY: Sí, solo esto (señalando una mitad). \\
6 & Inv1: ¿Por qué la hiciste así? \\
7 & NoY: Porque es así, como esta (señalando la mitad dada). \\
8 & Inv1: ¿Tú crees que está bien hecho? \\
9 & NoY: Si. \\
10 & Inv1: Vamos a ver (coge espejo de metacrilato). (Figura 3 izquierda). \\
11 & NoY: Sí. \\
12 & Inv2: ¿Y qué le pasa, a la luna que dibujaste y a la que se ve ahí? \\
13 & NoY: Que no está mucho doblada. \\
14 & Inv1: ¿Quieres dibujarla toda ahora? \\
15 & NoY: Vale (Figura 4 izquierda). \\
16 & NoY: Ya está (Figura 4 derecha). \\
17 & Inv2: Fíjate ahora, ¿qué ocurre? \\
18 & NoY: Son iguales los dos lados. \\
19 & Inv2: Si la doblas por aquí (señalando eje de simetría), ¿qué ocurre? \\
20 & NoY: Que este lado está contra este. \\
\hline
\end{tabular}

Figura 3 izquierda - La luna; Figura 3 derecha - Comprobación del dibujo.

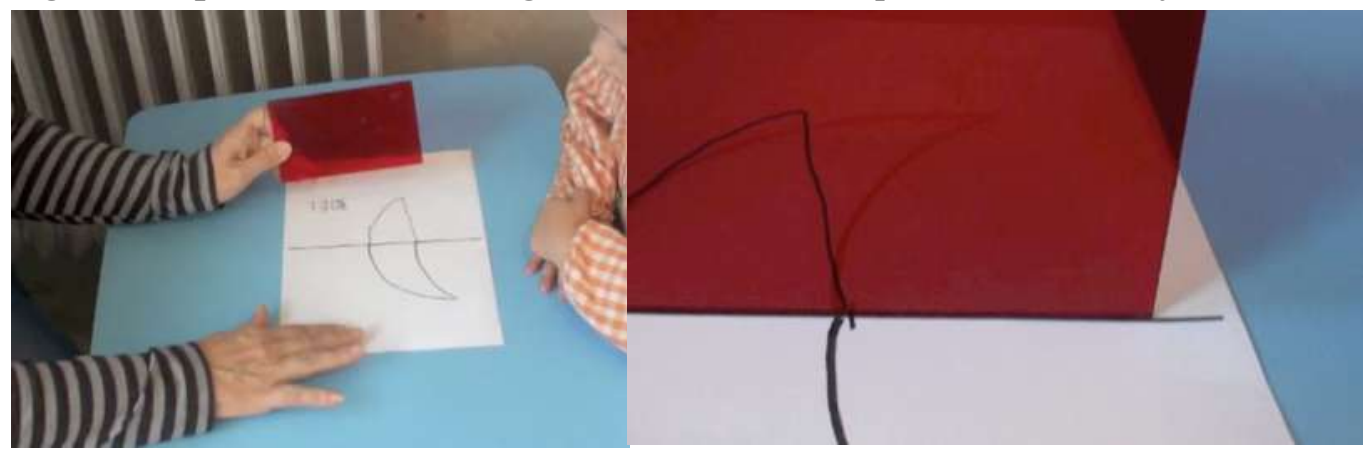

Fuente: archivo de la investigación 
Figura 4 izquierda - Modificación del dibujo; Figura 4 derecha - Resultado tras la comprobación.

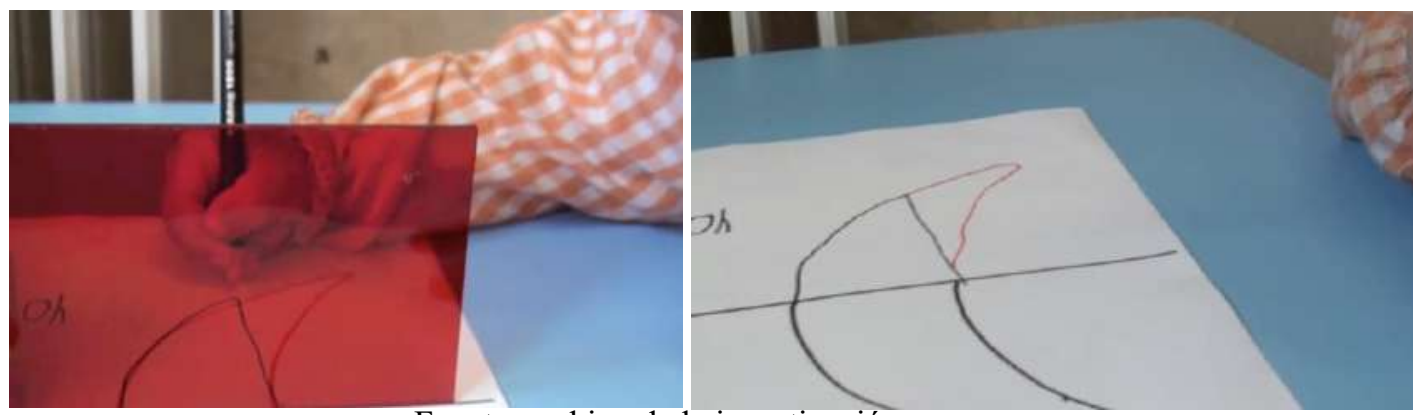

Fuente: archivo de la investigación

Al preguntar a este niño por qué hizo así el dibujo su respuesta es "porque es así como esta" (6), lo que pone de manifiesto que entiende que lo que se espera es una imagen simétrica a la dada. De sus respuestas, además, responde que cree que el dibujo está bien hecho. Al realizar la comprobación (Figura 3 derecha) ve la diferencia e intenta explicarla. También es capaz de reconocer la simetría - "son iguales los dos lados" (18) - y el eje “si la doblas por aquí ¿qué ocurre?” (19) “Que este lado está contra este” (20) - cuando observa el dibujo final (Figura 4), así como de justificarla.

\section{Actividad 7. Me sabe a...}

Se retoma el cuento ¿A qué sabe la luna?, y se plantea una asamblea en gran grupo sobre a qué le sabe a cada animal protagonista del cuento.

Entre toda la clase se opina y vota, llegando a acuerdos. Cada uno de los alimentos que aparece en la discusión es observado y analizado. Se formulan hipótesis sobre el eje o ejes de simetría, se dibuja el alimento en la pizarra y los estudiantes trazan los supuestos eje o ejes. A continuación, se manipulan los alimentos, haciendo los cortes y comprobando las hipótesis iniciales.

Con esta actividad se pretende evaluar la capacidad desarrollada por las niñas y los niños para descubrir la simetría presente en algunos alimentos y para identificar los ejes de simetría presentes, así como el modo en que justifican la presencia o no de simetría.

Tabla 6 - Diálogo de la actividad 7

\begin{tabular}{|l|l|}
\hline 1 & Inv2: Qué bien, ¿y los plátanos?, son como... \\
2 & NoRu: Una luna. \\
3 & Inv2: ¿Y son simétricos? \\
4 & NoRu: Sí. \\
5 & Inv2: Ven a dibujarlo... ¿por dónde pasarías el eje? \\
\hline
\end{tabular}




\begin{tabular}{|l|l|}
\hline 6 & NoRu: Así (Figura 5 izquierda). \\
7 & Inv2: Eso no es una línea recta... \\
8 & Inv2: Ven Yara... ilo dibujas?, el eje... (Figura 5 derecha). \\
9 & Inv2: Genial, coge el espejo para comprobarlo. Comprueba si es simétrico el \\
& plátano. \\
\hline
\end{tabular}

Figura 5 izquierda - Eje vertical fallido; Figura 5 derecha - Eje horizontal del plátano

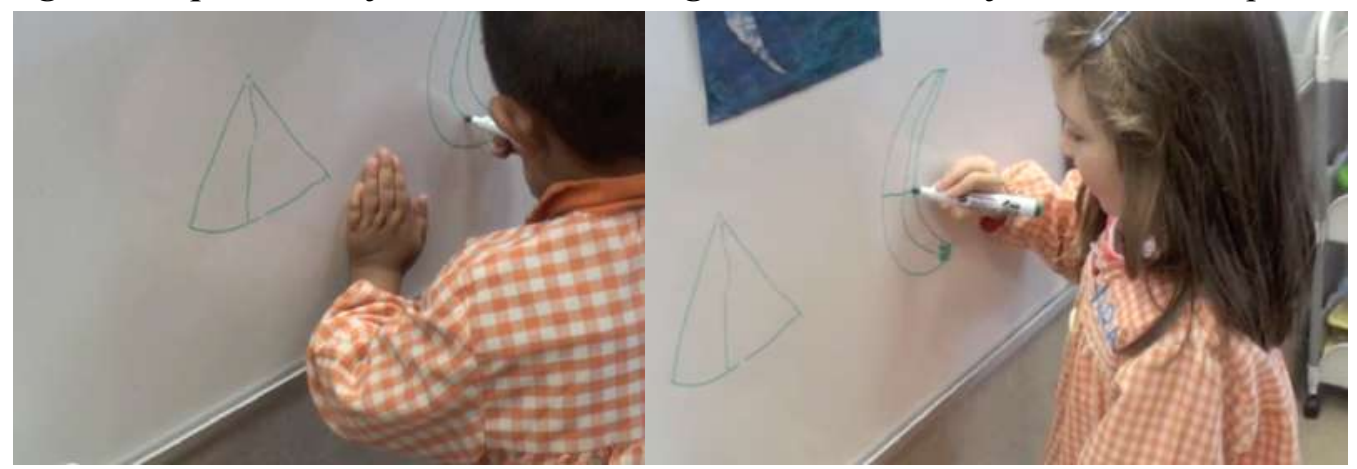

Fuente: archivo de la investigación

En las intervenciones de niños y niñas se observa que su primera respuesta trata de buscar un eje de simetría vertical, aunque en la representación plana del objeto de que se trata (en este caso un plátano) no existe este eje. Cuando se les hace ver esta circunstancia, enseguida caen en la cuenta del eje horizontal de simetría, que le recuerda al trabajado en la actividad de dibujo.

Tabla 7 - Simetría horizontal y vertical

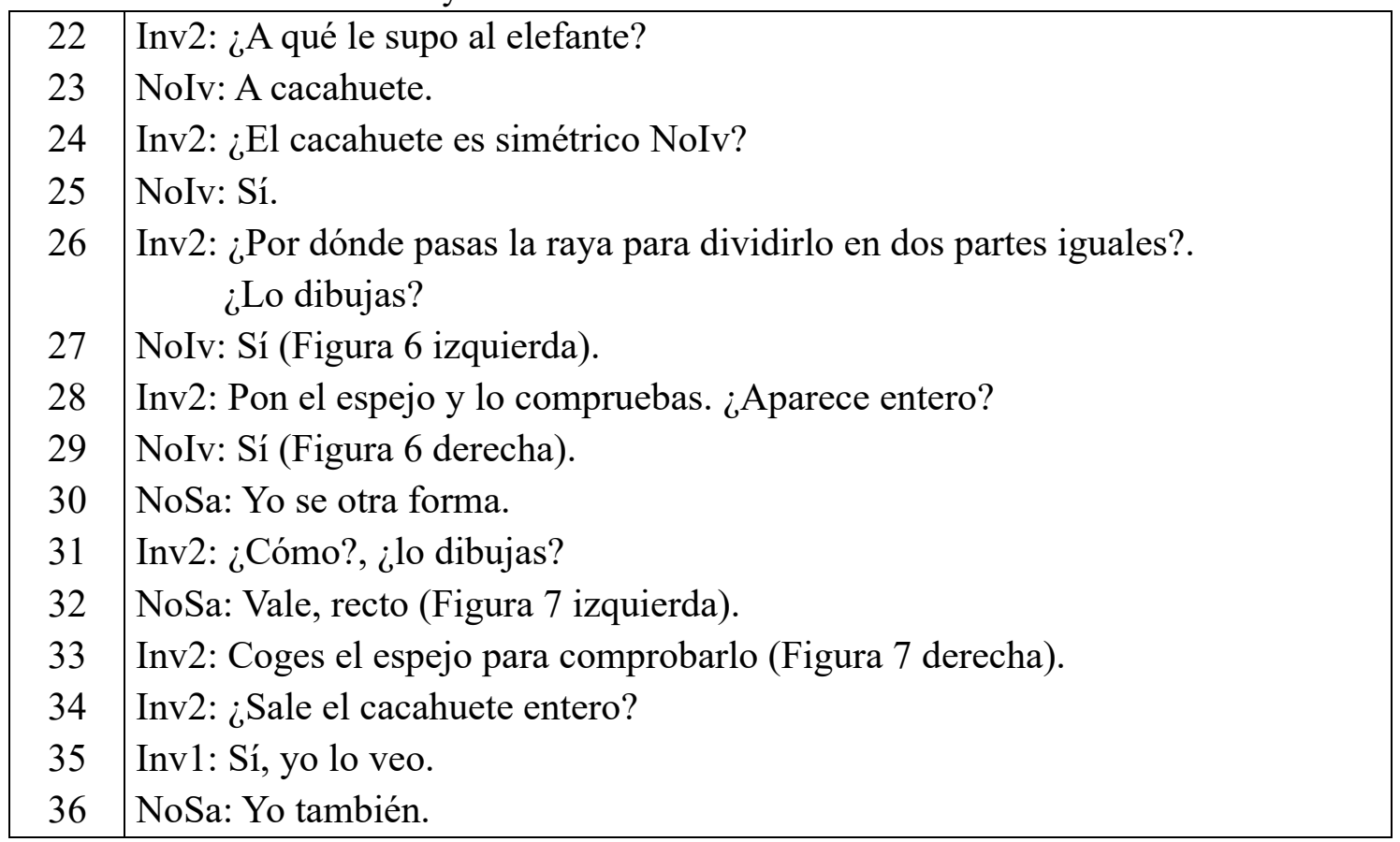


Figura 6 izquierda - Dibujo del eje horizontal; Figura 6 derecha - Comprobación del eje
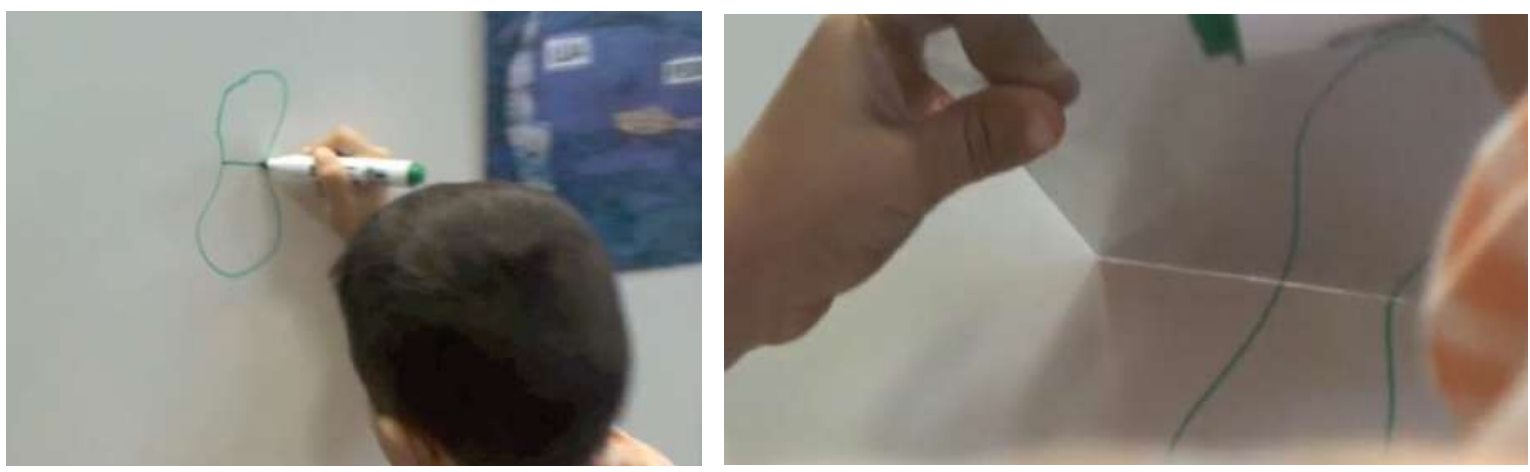

Fuente: archivo de la investigación

En este caso, el alimento dibujado tiene más de un eje de simetría. El primer eje detectado es el horizontal (Figura 6), pero una vez dibujado quieren representar también el eje vertical (Figura 7).

Figura 7 izquierda - Dibujo eje vertical; Figura 7 derecha - Comprobación del eje
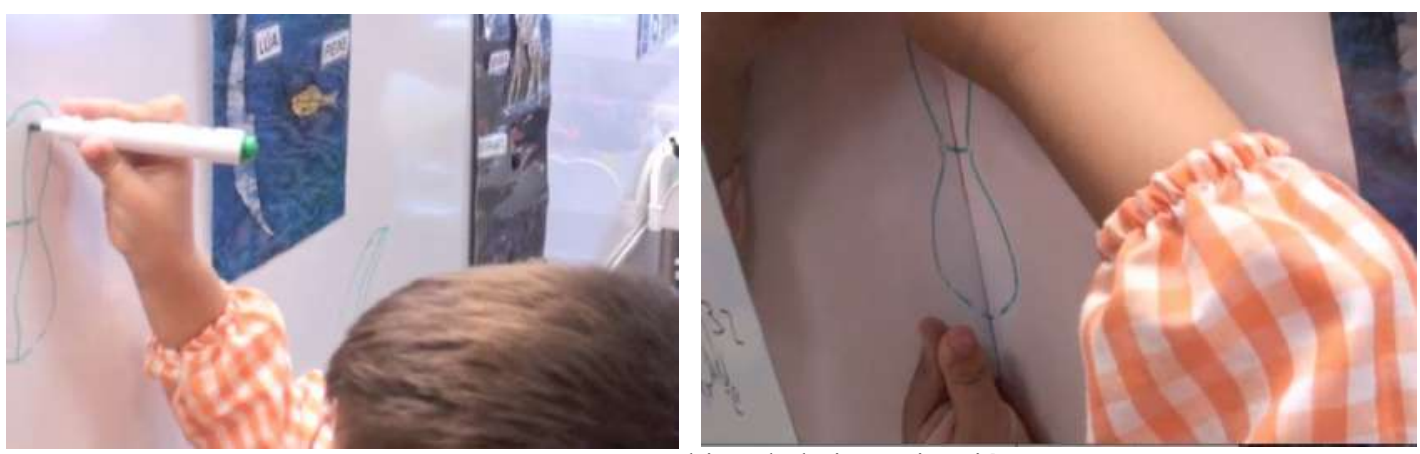

Fuente: archivo de la investigación

Para finalizar la indagación se presentan frutos redondos, con más de dos ejes de simetría que se descubren en la conversación.

Tabla 8 - Varios ejes de simetría

\begin{tabular}{|c|c|}
\hline 43 & Inv2: Dice NoSa, que la naranja que le gusta a la cebra es simétrica. ¿Qué más? \\
\hline 44 & $\begin{array}{l}\text { NoSa: Lo puedo demostrar. Se puede dividir en dos. Y en cuatro. } \\
\text { Y en seis así (Traza en el aire una línea inclinada). }\end{array}$ \\
\hline 45 & Inv2: ¿Cómo?, Sal, dibuja una naranja y divídela en 6 partes iguales. \\
\hline 46 & $\begin{array}{l}\text { NoSa: Así dos (traza línea vertical), así cuatro (traza línea horizontal), así seis } \\
\text { (traza línea oblicua) (Figura 8). }\end{array}$ \\
\hline 47 & Inv2: ¿Y ahora?, ¿son todas iguales? \\
\hline 48 & NoSa: Cogemos el espejo para comprobarlo. \\
\hline
\end{tabular}


Figura 8 - Ejes de simetría múltiples

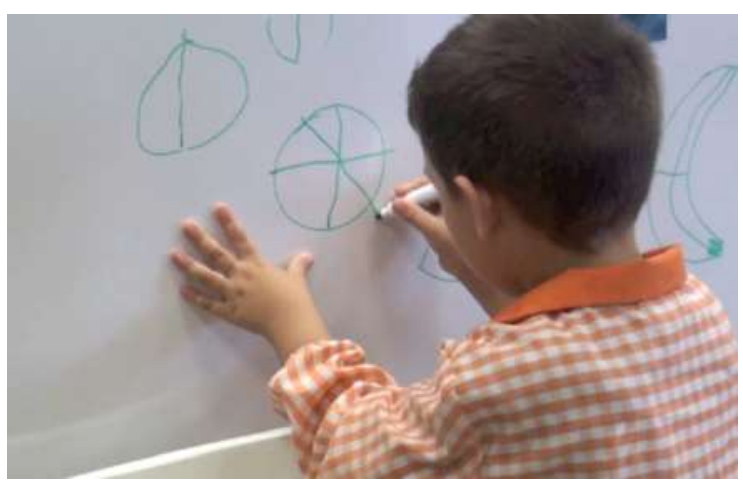

Fuente: archivo de la investigación

El reconocimiento e identificación del eje o ejes de simetría aparece de forma progresiva. Los niños y las niñas señalan primero un eje, que normalmente cuando existe es el vertical, y si no existe tal eje se plantean la búsqueda de otros. La experiencia con objetos que tienen más de un eje propicia que no se limiten a señalar el primero que ven, sino que busquen algún otro eje como puede ser el horizontal o ejes oblicuos. En el caso de los objetos circulares aparecen varias posibilidades, en general asociadas al número de partes en las que quieren dividir el objeto.

\section{Resultados relativos al proceso de descubrimiento y generación de simetrías}

Respecto a las dificultades surgidas en el proceso de descubrimiento y generación de simetrías podemos decir que, una vez descubierta la idea de simetría mediante la aparición contextualizada en el cuento del reflejo de la luna en el agua y de sus propias imágenes en el espejo de la clase, las niñas y los niños son capaces de reconocer visualmente las simetrías y discriminar figuras simétricas de otras que no lo son. La mayor parte del alumnado reconoce la simetría bilateral que se da a partir del eje de simetría, y la entiende como una transformación isométrica, que un objeto se repite, conservando forma y tamaño.

Sin embargo, en el momento de la generación no son conscientes de la necesidad de mantener las distancias respecto al eje de simetría de los puntos correspondientes a la hora de generar la mitad simétrica de una dada, ni de la condición de perpendicularidad al eje que han de mantener los segmentos que unen los puntos correspondientes. Debido a esta dificultad, sus producciones solamente se aproximan a lo que sería un resultado simétrico. 


\section{Resultados relativos a la identificación de los ejes de simetría}

En cuanto a la identificación de los ejes de simetría, hemos observado que los niños y las niñas son capaces de identificar un eje de simetría, máxime cuando es único o está claramente marcado, y en la mayor parte de los casos reconocen cuando el objeto analizado tiene más de uno.

Entre las dificultades observadas a la hora de identificar el eje de simetría aparece el dibujo de un eje de simetría no recto, marcando la línea central del objeto dibujado cuando es curvo. Esto parece deberse a la idea, correcta por otra parte, de que el eje divide el objeto por la mitad, si bien no tiene en cuenta la necesidad de que sea una línea recta. Esta dificultad se solventa mediante la comprobación, usando un espejo, de si el objeto es simétrico o no.

Otra dificultad detectada se produce cuando el objeto analizado tiene varios ejes de simetría, como es el caso de los objetos redondos. En estos casos la repuesta a la pregunta de cuál es el eje de simetría empieza siendo una línea vertical y a la que tras una reflexión se van sumando más ejes, aunque parece que les produce una cierta inseguridad el hecho de ver tantas posibilidades.

\section{Resultados relativos a la justificación de simetrías}

Respecto a las dificultades que aparecen en la justificación de la simetría, la más destacable es la falta de vocabulario adecuado para la institucionalización del conocimiento. Son capaces de describir las figuras simétricas y justificar la simetría con expresiones del tipo "porque esto es igual que esto" pero no son conscientes de las propiedades de equidistancia o perpendicularidad al eje, ni disponen del vocabulario preciso para expresarlas.

\section{Resumen de logros y dificultades a lo largo de la experimentación}

La Tabla 9 recoge de modo resumido, asociados a cada objetivo instruccional, tanto los logros alcanzados como las dificultades observadas a lo largo del experimento de enseñanza. 
Tabla 9 - Logros alcanzados y dificultades observadas

\begin{tabular}{|l|l|l|}
\hline $\begin{array}{l}\text { Objetivo } \\
\text { instruccional }\end{array}$ & Logro & Tipo de dificultad \\
\hline $\begin{array}{l}\text { Descubrimiento } \\
\text { de simetrías }\end{array}$ & $\begin{array}{l}\text { Reconocimiento visual de la } \\
\text { simetría (Act. 3, Act. 4, Act. 6) }\end{array}$ & $\begin{array}{l}\text { No reconoce la igualdad de distancias } \\
\text { al eje de puntos simétricos }\end{array}$ \\
\hline $\begin{array}{l}\text { Generación de fe } \\
\text { simetrías }\end{array}$ & $\begin{array}{l}\text { Dibujo de media figura } \\
\text { simétrica (Act. 5) }\end{array}$ & $\begin{array}{l}\text { No respeta la equidistancia respecto al } \\
\text { eje } \\
\text { No cumple la perpendicularidad }\end{array}$ \\
\hline $\begin{array}{l}\text { Identificación de } \\
\text { ejes de simetría }\end{array}$ & $\begin{array}{l}\text { Reconocimiento del eje de de } \\
\text { simetría } \\
\text { Identificación de más de un eje } \\
\text { (Act. 7) }\end{array}$ & $\begin{array}{l}\text { Dibujo de un eje no recto } \\
\text { Líneas que no son eje de simetría } \\
\text { Dificultad con la idea de que pueda } \\
\text { haber más de un eje }\end{array}$ \\
\hline $\begin{array}{l}\text { Justificación de la } \\
\text { simetría }\end{array}$ & $\begin{array}{l}\text { Descripción de figuras } \\
\text { simétricas } \\
\text { Justificación de la simetría } \\
\text { (Act. 6, Act. 7) }\end{array}$ & $\begin{array}{l}\text { Falta de vocabulario adecuado } \\
\text { Justificación visual, no basada en } \\
\text { equidistancia a al eje } \\
\text { perpendicularidad o }\end{array}$ \\
\hline
\end{tabular}

\section{CONCLUSIONES}

El desarrollo de experimentos de enseñanza en educación matemática permite establecer conclusiones sobre los aspectos destacables del aprendizaje (acorde con BERNABEU; MORENO; LLINARES, 2019). En el marco de la Educación Infantil permite recabar información relevante acerca de los procesos de aprendizaje que se producen en las primeras edades. Consideramos fundamental la implicación de la maestra en el desarrollo de este tipo de tareas para poder realizar un correcto enfoque de la investigación.

En este caso concreto, el enfoque desde una matemática contextualizada ha permitido acercar a niñas y niños un concepto, la simetría, que de otro modo podría resultar ajeno o demasiado abstracto. Al plantear las actividades enmarcadas en ámbitos tan cercanos como el cuento de partida o su propio cuerpo se ha fomentado su interés por descubrir simetrías en los objetos que les rodean.

El uso de los espejos como elemento auxiliar para la verificación de los resultados ha dado lugar a conversaciones en las que niñas y niños explican sus aciertos y justifican sus errores, lo que se traduce en un aprendizaje significativo.

Así, a lo largo del experimento de enseñanza hemos podido constatar cuáles son las dificultades que muestran los niños y las niñas de estas edades cuando se trabajan actividades dirigidas a la comprensión del concepto de simetría, pero también hemos 
podido detallar sus logros en las distintas fases. Entre los hallazgos, es claro que los niños y las niñas de estas edades muestran una mayor facilidad en la resolución de tareas que involucran la simetría vertical frente a aquellas que requieren el uso de la simetría horizontal (acorde con BORNSTEIN; FERDINANDSEN; GROSS, 1981). Además, les resulta más difícil detectar todos los ejes de simetría cuando el objeto dado contiene más de uno. Sin embargo, el uso de tareas contextualizadas ha provocado un interés en la búsqueda de todos los posibles ejes de simetría, dando lugar al descubrimiento de que hay figuras simétricas que no tienen por qué tener un eje de simetría vertical, y a la necesidad de justificación de por qué un objeto es simétrico o no. Todo ello, nos permite concluir que este experimento de enseñanza ha favorecido un acercamiento más natural al concepto de simetría.

Finalmente, con respecto a las limitaciones de la investigación debemos destacar el tipo de investigación, un estudio de casos, que, junto con el tamaño muestral (pequeño), no permite generalizar las conclusiones aquí mostradas.

Aun así, consideramos que la experimentación implementada, en la que se ha priorizado partir de las intuiciones e intereses de los niños y las niñas, ha dado lugar a trabajar de modo contextualizado y significativo el concepto de simetría, aspecto fundamental para poder trabajar la capacidad espacial (acorde a CLEMENTS, 2004).

\section{REFERÊNCIAS}

ACUÑA, C.; MARTÍNEZ, A.E. El aprendizaje de la reflexión en geometría entre estudiantes de primaria. In: CIAEM (ed.). Memorias XII CIAEM. Querétaro, México: CIAEM, 2013. p. 1-8.

ALSINA, A. Educación matemática en contexto de 3 a 6 años. Barcelona: ICE-Horsori, 2011.

ALSINA, A. Diseño, gestión y evaluación de actividades matemáticas competenciales en el aula. Épsilon-Revista de Educación Matemática, v. 33 (1), n. 92, p. 7-29, 2016.

ALSINA, A. La educación matemática infantil en España: ¿qué falta por hacer?.

Números-Revista de Didáctica de las Matemáticas, n. 100, p. 187-192, 2019.

ALSINA, A.; BERCIANO, A. Developing informal mathematics in early childhood education. Early Child Development and Care, v. 190, n.13, p. 2013-2031, 2020. http://doi.org/10.1080/03004430.2018.1555823

BERNABEU, M.; MORENO, M. Y LLINARES, S. Experimento de enseñanza como una aproximación metodológica a la investigación en Educación Matemática. Uni- 
Berciano, A.; Jiménez-Gestal, C.; Salgado, M.

pluriversidad, v. 19, p. 2, p.103-123, 2019.

BLANCO, T.F.; GORGAL, A.; SALGADO, M. Simetrías: frutas, vegetales y cuentos.

Épsilon-Revista de Educación Matemática, ESPAÑA, n. 98, p. 41-48, 2018.

BOE (2008). Boletín Oficial del Estado, de 19 de diciembre. Orden ECI/3960/2007.

BORNSTEIN, M. H.; FERDINANDSEN, K.; GROSS, C. G. Perception of symmetry in infancy. Developmental Psychology, v. 17, n. 1, p. 82-86, 1981.

https://doi.org/10.1037/0012-1649.17.1.82

BORNSTEIN, M. H.; STILES-DAVIS, J. Discrimination and memory for symmetry in young children. Developmental Psychology, v. 20, n. 4, p. 637- 649, 1984.

https://doi.org/10.1037/0012-1649.20.4.637

CANALS, M. A. La Geometría en las primeras edades escolares. Suma: Revista para la enseñanza y el aprendizaje de las matemáticas, n. 25, p. 31-44, 1997.

CLEMENTS, D. H. Geometric and Spatial Thinking in Early Childhood Education. In: CLEMENTS, D. H.; SARAMA, J; DIBIASE, A. M. Engaging young children in mathematics: Standards for Early Childhood Mathematics Education. Mahwah, NJ: Lawrence Erlbaum Associates, 2004. p. 267-297.

CLEMENTS, D. H. Major Themes and Recommendations. In: CLEMENTS, D. H.; SARAMA, J; DIBIASE, A. M. Engaging young children in mathematics: Standards for Early Childhood Mathematics Education. Mahwah, NJ: Lawrence Erlbaum Associates, 2004. p. 7-77.

FREUDENTHAL, H. Revisiting mathematics education. Dordrectht, Países Bajos: Kluwer Academic. 1991.

GENKINS, E. F. The concept of bilateral symmetry in young children. In: ROSSKOPF, M. F. (Ed.), Children's mathematical concepts: Six Piagetian studies in mathematics education New York: Teachers College Press, 1975. p. 5-43.

GIMÉNEZ, J.; VANEGAS, Y. Contextualizações de transformações geométricas na Educação Infantil. Perspectivas da Educação Matemática, v. 12, n. 28, p. 56-73, 2019.

GODINO, J. D.; GONZATO, M.; CAJARAVILLE, J. A.; FERNÁNDEZ, T. Una aproximación ontosemiótica a la visualización en educación matemática. Enseñanza de las Ciencias, v. 30, n. 2, 109-130, 2012.

HIDAlGO, S.; MAROTO, A.; ORTEGA, T.; PALACIOS, A. Atribuciones de Afectividad hacia las Matemáticas. Unión: Revista Iberoamericana de Educación Matemática, n. 35, 93-113, 2013.

JIMÉNEZ-GESTAL, C.; BERCIANO, A.; SALGADO, M. Cómo trabajar la orientación espacial de modo significativo en Educación Infantil: implicaciones didácticas. Revista de Educación Matemática, v. 3, n. 2, p. 61-74, 2019. DOI: 10.24844/EM3102.03

MINISTERIO DE EDUCACIÓN Y CIENCIA (España). Orden ECI/3963/2007, de 19 de diciembre. Por la que se establece el currículo y se regula la ordenación de la educación infantil. Boletín Oficial del Estado: 222, Madrid, n. 5, p. 1016-1036, 5 enero. 2008.

MOLINA, M.; CASTRO, E.; MOLINA, J.L.; CASTRO, E. (2011). Un acercamiento a la

ReviSeM, Ano 2021, N. 2, p. $24-46$ 
investigación de diseño a través de los experimentos de enseñanza. Enseñanza de las Ciencias, v. 29, n. 1, p. 75-88, 2011.

NCTM. Principios y estándares para la educación matemática. Sevilla, España: SAEM Thales. 2003.

NOVO, M. L.; BERCIANO, A.; ALSINA, A. (2019). Conexiones matemáticas de tipo conceptual en niños de 4 años. REDIMAT - Journal of Research in Mathematics Education, v. 8, n. 2, p. 166-192. DOI: 10.4471/redimat.2019.3938

OCDE. Marco de Evaluación y de Análisis de PISA para el Desarrollo: Lectura, matemáticas y ciencias, Versión preliminar. Paris, Francia: OECD Publishing, 2017.

SALGADO, M.; BERCIANO, A.; JIMÉNEZ-GESTAL, C. Aproximación a los objetos tridimensionales y sus propiedades en el aula de educación infantil: una experiencia de aula con cilindros. Unión, n. 54, p. 46-59, 2018.

SÁMUEL, M.; VANEGAS, Y.; GIMÉNEZ, J. Visualización y simetría en la formación de maestros en Educación Infantil. Edma 0-6: Educación Matemática en la Infancia. v. 5, n.1, p. 21-32, 2016.

SARAMA, J.; CLEMENTS, D.H. Early Childhood Mathematics Education Research: Learning Trajectories for Young Children. 1. ed. New York: Routledge, 2009.

SARAMA, J.; CLEMENTS, D. H.; VUKELIC, E. B. The role of a computer manipulative in fostering specific psychologica $/$ mathematical processes. In: E. JAKUBOWSKI, D. WATKINS, \& H. BISKE (Eds.), Proceedings of the 18th annual meeting of the North America Chapter of the International Group for the Psychology of Mathematics Education. Columbus, OH: ERIC Clearinghouse for Science, Mathematics, and Environmental Education, 1996. v. 2, p. 567-572.

VURPILLOT, E. The visual world of the child. International U Press, 1976. 